\title{
Efecto del extracto acuoso, ácido y alcohólico de las hojas secas de Erythroxylum coca var coca (coca) en Trichophyton rubrum, Trichophyton mentagrophytes, Microsporum canis y Candida albicans in vitro
}

\author{
Medalit Luna-Vílchez ${ }^{1}$, Cristian Díaz-Vélez², Francisco Baca-Dejo ${ }^{3}$
}

RESUMEN

Objetivo: Evaluar el efecto del extracto acuoso, ácido y alcohólico de las hojas secas Erythroxylum coca var coca (coca) en Trichophyton rubrum (TR), Trichophyton mentagrophytes (TM), Microsporum canis (MC) y Candida albicans (CA) in vitro.

Materiales y métodos: Estudio experimental, que evaluó la presencia o ausencia de crecimiento, la velocidad de crecimiento de los hongos a partir del punto de siembra comparando con su crecimiento en Agar Sabouraud (AS). Se analizó con Siegel-Tukey, Kruskal-Wallis, con $\mathrm{p}<0,05$.

Resultados: El grupo CA encontró diferencia estadística $(p<0,05)$ entre grupos etanol $99.9 \%$ vs Acuoso, etanol $99.9 \%$ vs $\mathrm{HCl} 0.01 \mathrm{M}$, etanol $99.9 \%$ vs control, control vs acuoso, $\mathrm{HCl} 0.01 \mathrm{M}$ vs control, el grupo TR encontró diferencia ( $\mathrm{p}<0,05)$ entre los grupos etanol $99.9 \%$ vs $\mathrm{HCl} 0.01 \mathrm{M}$, etanol $99.9 \%$ vs acuoso, etanol $99.9 \%$ vs control, $\mathrm{HCl} 0.01 \mathrm{M}$ vs control; el grupo TM encontró diferencia $(\mathrm{p}<0,05)$ entre los grupos: etanol $99.9 \%$ vs $\mathrm{HCl} 0.01 \mathrm{M}$, etanol $99.9 \%$ vs control, acuoso vs ácido, acuoso vs control.; grupo $\mathrm{MC}$ encontró diferencia $(\mathrm{p}<0,05)$ entre los grupos: etanol $99.9 \% \mathrm{vs} \mathrm{HCl} 0.01 \mathrm{M}$, etanol 99.9\% vs control, acuoso vs ácido, acuoso vs control. En todos los casos el grupo HCl $0.01 \mathrm{M}$ vs acuoso fue no significativo. Conclusiones: Los extractos acuoso, ácido y alcohólico no presentan efecto sobre el crecimiento de CA y TM pero sí sobre TR y MC en extracto alcohólico. Además hubo diferencia de velocidad de crecimiento de CA, TR y TM en extractos acuoso, ácido y alcohólico comparado con AS, pero entre ellos solo TR, TM y MC presentaron diferencia con la velocidad.

Palabras clave: Coca; micosis; medicina tradicional; planta medicinal.

\section{Effect of the aqueous, acidic and alcoholic extract of dried leaves of Erythroxylum coca var. coca (coca) in Trichophyton rubrum, Trichophyton mentagrophytes, Microsporum canis and Candida albicans in vitro}

\begin{abstract}
Objective: To evaluate the effect of the aqueous, acidic and alcoholic extract of dried leaves of Erythroxylum coca var. coca (coca) in Trichophyton rubrum (TR), Trichophyton mentagrophytes (TM), Microsporum canis (MC) and Candida albicans (CA) in vitro.

Materials and methods: An experimental study which evaluated the presence or absence of fungal growth, and fungal growth rate from the seeding point, compared to fungal growth in Sabouraud Agar (SA) using Siegel-Tukey and KruskalWallis tests $(p<0.05)$.

Results: The CA group showed a statistical difference $(\mathrm{p}<0.05)$ between the following groups: $99.9 \%$ ethanol vs. aqueous, $99.9 \%$ ethanol vs. $0.01 \mathrm{M} \mathrm{HCl}, 99.9 \%$ ethanol vs. control, control vs. aqueous, $0.01 \mathrm{M} \mathrm{HCl}$ vs. control. The TR group showed a statistical difference $(\mathrm{p}<0.05)$ between the following groups: $99.9 \%$ ethanol vs. $0.01 \mathrm{M} \mathrm{HCl}, 99.9 \%$ ethanol vs. aqueous, 99.9\% ethanol vs. control, $0.01 \mathrm{M} \mathrm{HCl}$ vs. control. The TM group showed a statistical difference $(\mathrm{p}<0.05)$ between the following groups: $99.9 \%$ ethanol vs. $0.01 \mathrm{M} \mathrm{HCl}, 99.9 \%$ ethanol vs. control, aqueous vs. acid, aqueous vs. control. The $\mathrm{MC}$ group showed a statistical difference $(p<0.05)$ between the following groups: $99.9 \%$ ethanol vs. $0.01 \mathrm{M} \mathrm{HCl}, 99.9 \%$ ethanol vs. control, aqueous vs. acid, aqueous vs. control. In all the cases, the results of the $0.01 \mathrm{M} \mathrm{HCl}$ vs. aqueous group were not significant.

Conclusions: The aqueous, acidic and alcoholic extracts have no effect on the growth of CA and TM, but the alcoholic extract has effects on the growth of TR and MC. In addition, there were differences in the growth rate of CA, TR and TM in the aqueous, acidic and alcoholic extracts compared to that in SA. However only TR, TM and MC showed differences in their growth rate in the alcoholic extract.
\end{abstract}

Keywords: Coca; mycoses; traditional medicine; medicinal plant.

1. Médico Cirujano, Universidad de San Martín de Porres - Filial Norte. Chiclayo, Perú.

2. Médico Epidemiólogo, Hospital Nacional Almanzor Aguinaga Asenjo-EsSalud. Chiclayo, Perú.

3. Biólogo - Microbiólogo, Universidad de Chiclayo. Chiclayo, Perú. 


\section{INTRODUCCIÓN}

Las infecciones por hongos en la piel y las uñas son un problema mundial común, siendo una de las infecciones más frecuentes, llegando a tener una prevalencia las micosis superficiales de $20-25 \%$ en la población mundial siendo los patógenos responsables principalmente dermatofitos antropofílicos y zoofílicos de los géneros Trichophyton T., Microsporum M. y Epidermophyton E. ${ }^{(1)}$.

Las micosis superficiales son aquellas en las que hay afección de la capa córnea de la piel y la porción suprafolicular del pelo. Estas se dividen en: piedras (Trichosporum beigelii y Piedraia hortai), pitiriasis y dermatitis seborreica (levaduras del género Malassezia) y tínea nigra (Phaeoannellomyces werneckii). Las micosis cutáneas afectan capas más profundas de la piel y sus anexos pelos y uñas; se dividen en: tineas o dermatofitosis (dermatofitos de los géneros Microsporum, Epidermophyton y Trichophyton) y candidiasis cutánea (levaduras del género Cándida) ${ }^{(2)}$.

En un estudio realizado en Laboratorio de Micología del Instituto de Medicina Tropical "Daniel Alcides Carrión", Universidad Nacional Mayor de San Marcos, correspondiente al periodo 1976-2005 donde el 55,3\% de las de pacientes con sospecha clínica de dermatomicosis resultaron positivos encontrándose a Trichophyton rubrum $(33,2 \%)$, Candida albicans $(15,3 \%)$, Candida no albicans $(11,8 \%)$, Trichophyton mentagrophytes $(9,4 \%)$, Malassezia spp $(9,1 \%)$ y las infecciones mixtas (casos en donde hubieron dos patógenos responsables) $(7,2 \%)$ fueron los principales agentes infecciosos encontrado ${ }^{(3)}$, y en un estudio realizado en dos departamentos (San Martín y Madre de Dios) encontraron mayor prevalencia de onicomicosis de mano, pitiriasis versicolor y tiña pedis entre 1.5\%-4.5\%; para onicomicosis de pie $6.2 \%$ y tiña de $9.5 \%$. El dermatofito más frecuentemente aislado, fue del género Trichophyton y los cuadros clínicos más frecuentes fueron onicomicosis, tiña Corporis y tiña pedís, mientras que en Onicomicosis de pie, el género Candida sp fue el más prevalente $(83 \%)^{(4)}$.

Hay muchos productos naturales de los que se pueden obtener sustancias de valor terapéutico con una mínima transformación. Las plantas, los hongos y los organismos marinos sésiles son las más fuentes más prometedoras. Los productos naturales han tenido usos terapéuticos desde la antigüedad y aún siguen usándolos cuatro de cada cinco habitantes ${ }^{(5)}$.

Es conocido el uso de la hoja de coca (del genero Erythroxylum) como manifestación del patrimonio cultural (6), para este género se señala el aislamiento de alcaloides y flavonoides como grupos químicos fundamentales, siendo las hojas y el tronco las partes más estudiadas de este género ${ }^{(7-10)}$. Entre los componentes descritos de la hoja de coca encontramos metabolitos primarios como proteínas, carbohidratos y lípidos; y metabolitos secundarios como alcaloides, flavonoides, taninos, glicósidos y aceite esencial (11-14), encontrándose una mayor presencia de alcaloides en el tronco, ramas y hojas y de flavonoides en la hoja de la planta, los cuales han mostrado una importante actividad biológica incluyendo antifúngica y antibacteriana ${ }^{(9,15-18)}$; y dada la importancia de los alcaloides se ha desarrollado diversos métodos para obtenerlos, lo cual no sorprende por la enorme variedad de estructuras que lo caracterizan, y el amplio rango de solubilidad y de otras propiedades que presentan; sin embargo entre ellos se aprovecha su carácter básico para extraerlos de la planta que los contiene usando soluciones acuosas o débilmente ácidas como el ácido clorhídrico $(\mathrm{HCl} 1 \mathrm{~N}){ }^{(19)}$.

Los flavonoides son otros de los grupos químicos que se registran más en la literatura científica para este género; estos se localizan principalmente en las hojas de las plantas

${ }^{(18)}$. Los flavonoides isolados han mostrado una importante actividad biológica incluyendo antifúngica y antibacterian ${ }^{(20,21)}$. Muchos mecanismos de acción de los diferentes flavonoides han sido propuestos incluyendo su acción inhibitoria de la síntesis de ácidos nucleicos, inhibición de la función de la membrana citoplasmática e inhibición de la energía del metabolismo ${ }^{(22)}$. Los solventes empleados en la extracción de estos compuestos son muy variados y pueden ser desde muy polares como el agua y etanol para glicósidos o aglicanos muy hidroxiladas, hasta menos polares como éter y cloroformo para flavonas altamente metoxiladas ${ }^{(19)}$.

Además debemos tomar en cuenta que existe una alta citotoxicidad durante los tratamientos prolongados por diversas drogas antifúngicas ${ }^{(23)}$, por lo que el uso de medios más naturales podrían ayudar a mayor adherencia a los tratamientos, por ello se tuvo como objetivo evaluar el efecto sobre el crecimiento microbiológico y la velocidad de crecimiento del extracto acuoso, ácido y alcohólico de la hoja seca de coca (Erythroxylum coca var coca) en Trichophyton rubrum, Trichophyton mentagrophytes, Microsporum canis y Cándida albicans in vitro.

\section{MATERIALES Y MÉTODOS}

\section{Diseño del estudio}

Estudio experimental, prospectivo, longitudinal.

\section{Material de plantas}

Se utilizaron cepas de Trichophyton rubrum (TR), Trichophyton mentagrophytes (TM), Microsporum canis (MC) y Cándida albicans (CA) las cuales fueron cultivadas en los extractos acuoso, ácido y alcohólico de hoja seca de coca (Erythroxylum coca var coca) (Tabla 1). 
Tabla 1. Nombres de los grupos de extractos y de las cepas de hongos

\begin{tabular}{|c|c|c|l|}
\multicolumn{2}{c|}{$\begin{array}{c}\text { Grupos de los } \\
\text { extractos }\end{array}$} & Abrev. & \multicolumn{1}{c|}{ Cepas } \\
\hline G1 & Control & CA & Candida albicans \\
\hline G2 & Acuoso & TR & Trichophyton rubrum \\
\hline G3 & HCl 0.01M & TM & Trichophyton mentagrophytes \\
\hline G4 & Etanol $99.9 \%$ & MC & Microsporum canis \\
\hline
\end{tabular}

Se obtuvieron los extractos mediante técnicas de molienda, cocción secado y mezcla. El secado de la planta a estudiar se realizó en una estufa a $37^{\circ} \mathrm{C}$, durante 24 horas, luego se sometió a una etapa de molienda, obteniéndose una muestra de 20 gramos de polvo fino de coloración verde.

\section{Preparación de los extractos acuoso, alcohólico y ácido}

Se pesó 1 gramo de la muestra en el matraz de Erlenmeyer, se agregó $10 \mathrm{ml}$. del solvente (agua destilada $\mathrm{pH}=7$ para el extracto acuoso, etanol 99,9\% para el extracto alcohólico, ácido clorhídrico al $1 \%$ para el extracto ácido) y se colocó en baño María durante 10 minutos, se procedió a enfriar y filtrar la muestra, preparando un total de 80 placas de las cuales 5 pertenecen a cada hongo por cada extracto incluyendo el Agar Sabouraud solo (grupo control).

\section{Medición del efecto}

Para medir el efecto microbiológico sobre el crecimiento las cepas fueron cultivadas en los extractos mencionados, observándose presencia o ausencia de crecimiento. La velocidad de crecimiento se obtuvo mediante medición del diámetro del crecimiento de los hongos a partir del punto de siembra comparando con su crecimiento en Agar
Sabouraud, este procedimiento se realizó durante un periodo de 20 días y en el cual se registró el crecimiento de los hongos a diario, usándose la fórmula de velocidad de crecimiento midiendo el diámetro final menos el diámetro inicial entre la distancia en milímetros.

\section{Análisis estadístico}

Se usó estadística descriptiva calculando frecuencias absolutas y relativas para las variables cualitativas y media, mediana, desviación estándar para las cuantitativas. Se utilizó además la prueba estadística de Siegel-Tukey, Kruskal-Wallis, para el análisis inferencial, usando el software estadístico para el análisis de datos SPSS versión 20, y Microsoft Excel para Windows con nivel de confianza de $95 \%$ y nivel de significancia estadística $\mathrm{p}<0,05$.

\section{Aspectos éticos}

No fue requerido el consentimiento informado para el siguiente trabajo debido a que no participaran seres humanos, sin embargo se solicitó permiso a la universidad San Martín de Porres-Filian Norte para la utilización de los ambientes y material a utilizar.

\section{RESULTADOS}

Tabla 2. Comparación intergrupos según tipo de tratamiento del crecimiento de Candida albicans, Trichophyton rubrum, Trichophyton mentagrophytes y Microsporum canis

\begin{tabular}{|c|c|c|c|c|c|c|c|c|c|c|c|c|}
\hline \multirow[t]{2}{*}{ Grupo ${ }^{\beta}$} & \multicolumn{3}{|c|}{ Candida albicans } & \multicolumn{3}{|c|}{$\begin{array}{l}\text { Trichophyton } \\
\text { rubrum }\end{array}$} & \multicolumn{3}{|c|}{$\begin{array}{l}\text { Trichophyton } \\
\text { mentagrophytes }\end{array}$} & \multicolumn{3}{|c|}{ Microsporum canis } \\
\hline & $\mathrm{x}$ & DS & $\mathbf{p}^{\alpha}$ & $\mathbf{x}$ & DS & $\mathbf{p}^{\alpha}$ & $\mathbf{X}$ & DS & $p^{\alpha}$ & $\mathbf{X}$ & DS & $\mathbf{p}^{\alpha}$ \\
\hline Control & 2,68 & 0,67 & & 0,87 & 0,47 & & 1.65 & 0.75 & & 15.26 & 8.73 & \\
\hline Acuoso & 1,53 & 0,21 & $p<0,05$ & 0,64 & 0,28 & $p<0,05$ & 0.9 & 0.43 & $p<0,05$ & 2.09 & 1.13 & $p<0,05$ \\
\hline $\mathrm{HCl} 0.01 \mathrm{M}$ & 1,66 & 0,28 & & 0,53 & 0,2 & & 0.83 & 0.42 & & 13.56 & 8.01 & \\
\hline $\begin{array}{l}\text { Etanol } \\
99.9 \%\end{array}$ & 1,03 & 0,43 & & 0 & 0 & & 0.37 & 0.27 & & 0 & 0 & \\
\hline
\end{tabular}

a:calculado con la prueba de Kruskal-Wallis.

B: Comparación intergrupos calculado con prueba de Tukey. 
El grupo de CA se encontró diferencia estadísticamente significativa $(\mathrm{p}<0,05)$ entre los grupos: etanol $99.9 \%$ vs Acuoso, etanol $99.9 \%$ vs $\mathrm{HCl} 0.01 \mathrm{M}$, etanol $99.9 \%$ vs control, control vs acuoso, $\mathrm{HCl} 0.01 \mathrm{M}$ vs control; solo no siendo significativo $(p>0,05)$ el grupo $\mathrm{HCl} 0.01 \mathrm{M}$ vs acuoso.

El grupo de TR se encontró diferencia estadísticamente significativa $(p<0,05)$ entre los grupos: etanol $99.9 \% \mathrm{vs} \mathrm{HCl}$ $0.01 \mathrm{M}$, etanol $99.9 \%$ vs acuoso, etanol $99.9 \%$ vs control, $\mathrm{HCl} 0.01 \mathrm{M}$ vs control; solo no siendo significativo $(p>0,05)$ el $\mathrm{HCl} 0.01 \mathrm{M}$ vs acuoso, acuoso vs control.
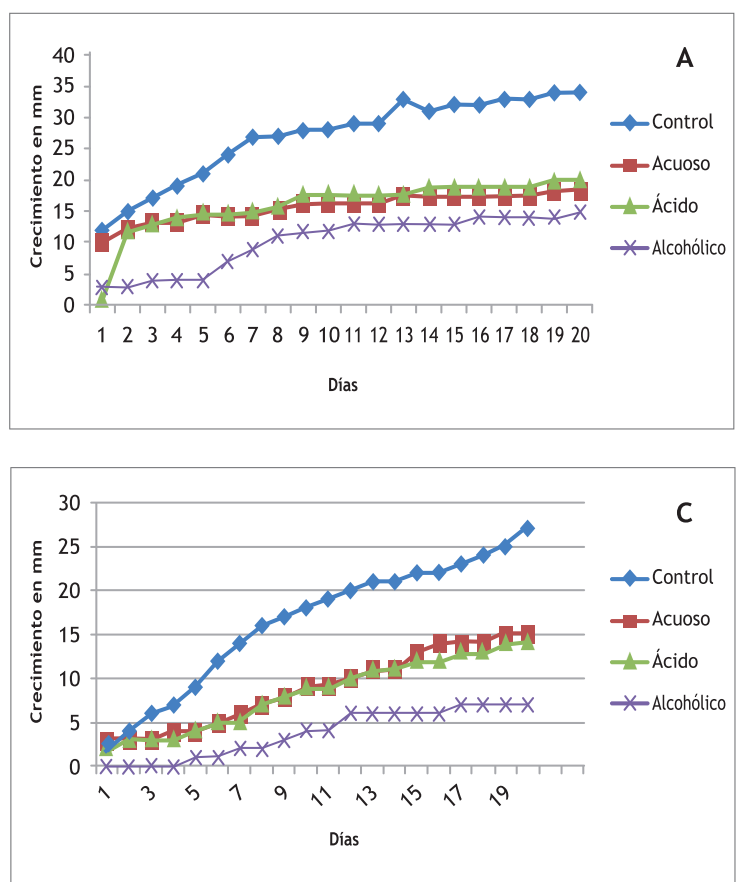

El grupo de TM se encontró diferencia estadísticamente significativa $(p<0,05)$ entre los grupos: etanol $99.9 \% \mathrm{vs} \mathrm{HCl}$ $0.01 \mathrm{M}$, etanol $99.9 \%$ vs control, acuoso vs ácido, acuoso vs control.; solo no siendo significativo $(p>0,05)$ el $\mathrm{HCl} 0.01 \mathrm{M}$ vs control.

El grupo de $M C$ se encontró diferencia estadísticamente significativa $(p<0,05)$ entre los grupos: etanol $99.9 \% \mathrm{vs} \mathrm{HCl}$ $0.01 \mathrm{M}$, etanol $99.9 \%$ vs control, acuoso vs ácido, acuoso vs control; solo no siendo significativo $(p>0,05)$ el $\mathrm{HCl} 0.01 \mathrm{M}$ vs control.
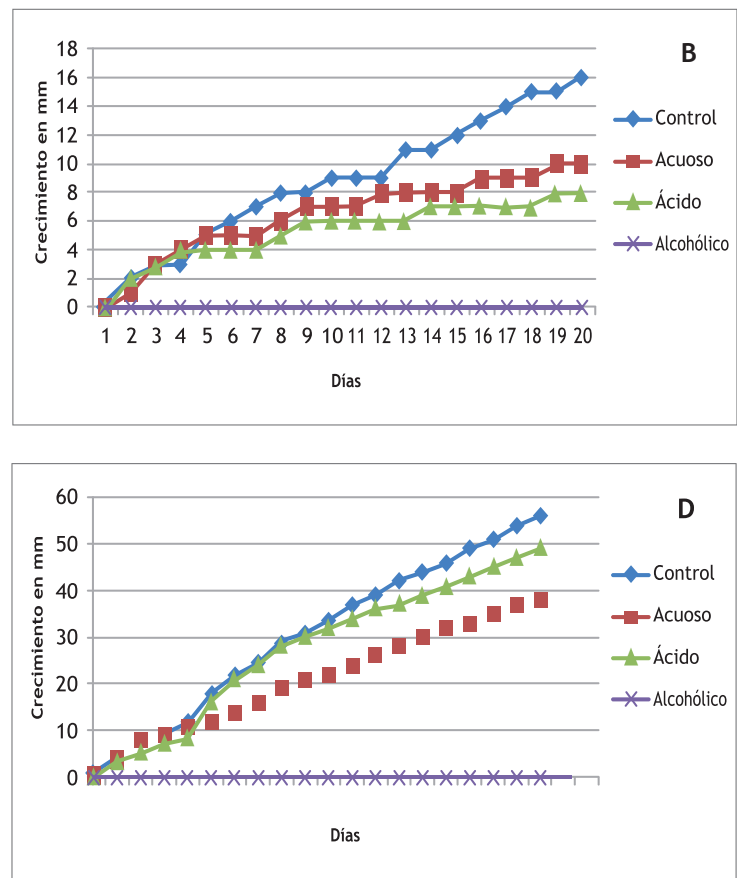

Figura 1. Velocidad de crecimiento en $\mathrm{mm}$ /día en Agar Sabouraud, extracto acuoso, extracto ácido y extracto alcohólico de hoja seca de coca (Erythroxylum coca var coca) in vitro de A: Candida albicans, B: Trichophyton rubrum, C: Trichophyton mentagrophytes y D: Microsporum canis.

\section{DISCUSIÓN}

Los extracto acuoso, ácido y alcohólico no presentaron efecto microbiológico sobre el crecimiento de Candida albicans, pero sí disminuyeron su velocidad de crecimiento comparado con el grupo control, además se encontró diferencia significativa del crecimiento microbiológico de Candida albicans en el extracto alcohólico comparado con el grupo control y los extractos acuoso y ácido, del extracto acuoso comparado con el grupo control y del extracto ácido comparado con el grupo control. Estos resultados difieren de los estudios de diversas plantas como Broussonetia papyrifera, Aegle marmelos, Piper crassinervium cuyos principales componentes alcaloides y flavonoides presentan efecto inhibitorio del crecimiento de Candida albicans, pudiendo ser debido a las mayores concentraciones utilizadas de las plantas en estos estudios ${ }^{(4,15,24-28)}$.
Los extractos acuoso y ácido no presentaron efecto microbiológico sobre el crecimiento de Trichophyton rubrum pero si lo presentó el extracto alcohólico, esto puedo ser debido a que el alcohol tiene mayor afinidad para la extracción de flavonoides el cual posee actividad antifúngica ${ }^{(19)}$, se observó además disminución de la velocidad de crecimiento en los tres extractos comparados con el control y se encontró diferencia significativa del crecimiento microbiológico de Trichophyton rubrum en el extracto alcohólico comparado con el grupo control y los extractos ácido y acuoso; y del extracto ácido comparado con el grupo control, así como lo demuestra García V. et al, donde menciona que las plantas mexicanas como Sedum spp. y Annona cherimolia cuyos principales componentes fueron los flavonoides y alcaloides respectivamente mostraron actividad antifúngica en el crecimiento de Trichophyton rubrum ${ }^{(26)}$. 
Los extractos acuoso, ácido y alcohólico no presentaron efecto microbiológico sobre el crecimiento de Trichophyton mentagrophytes pero si disminuyeron su velocidad de crecimiento comparado con el grupo control y se encontró diferencia significativa del crecimiento microbiológico de Trichophyton mentagrophytes en el extracto alcohólico comparado con el grupo control y los extractos ácido y acuoso, del extracto ácido comparado con el grupo control y del extracto acuoso comparado con el grupo control, similar a lo encontrado por Mishra et al y García V. et al, donde plantas en cuyos componentes se encontraron los flavonoides y alcaloides como Sedum spp. , Annona cherimolia y Aegle marmelos demostraron actividad antifúngica disminuyendo el crecimiento Trichophyton mentagrophytes ${ }^{(26,27)}$.

Sólo el extracto alcohólico presentó efecto microbiológico sobre el crecimiento de Microsporum canis, este resultado pudo ser debido a que el alcohol y el agua extraen los alcaloides y flavonoides en conjunto de la hoja de la planta en comparación con el ácido que tiene mayor afinidad por la extracción de los alcaloides. Se encontró diferencia significativa del crecimiento microbiológico de Microsporum canis en el extracto alcohólico comparado con el grupo control y el extracto ácido, del extracto acuoso comparado con el grupo control y el extracto ácido. No se observó diminución de la velocidad de crecimiento en los extractos comparados con el grupo control ${ }^{(19)}$.

Concluyéndose que los extractos acuoso, ácido y alcohólico de hoja seca de coca no presentan efecto sobre el crecimiento de CA y TM pero sí sobre el crecimiento de TR y MC en extracto alcohólico. Además hubo diferencia de velocidad de crecimiento de CA, TR y TM en extractos acuoso, ácido y alcohólico comparado con AS, pero entre ellos solo TR, TM y MC presentaron diferencia con la velocidad de crecimiento en el extracto alcohólico.

Agradecimientos: Empresa Nacional de la coca ENACO S.A. por habernos brindado las hojas de coca (Erythroxylum coca var coca) para el estudio. A la Dra. Beatriz Bustamante Rufino, jefe del laboratorio de Micología del Instituto de Medicina Tropical "Alexander Von Humbolt" por el apoyo brindado las cepas tipificadas de los hongos Trichophyton rubrum, Trichophyton mentagrophytes, Microsporum canis y Cándida albicans.

\section{REFERENCIAS BIBLIOGRÁFICAS}

1. Havlickova B, Czaika VA, Friedrich M. Epidemiological trends in skin mycoses worldwide. Mycoses. 2008;51(Suppl 4):2-15.

2. Nardin ME, Pelegri DG, Manias VG, Méndez E. Agentes etiológicos de micosis superficiales aislados en un Hospital de Santa Fe: Argentina. Rev Argent Microbiol. 2006;38(1):25-7.

3. Bejar V, Villanueva F, Guevara JM, González S, Vergaray G, Abanto $\mathrm{E}$, et al. Epidemiología de las dermatomicosis en 30 años de estudio en el Instituto de Medicina Tropical Daniel A Carrión, Universidad Nacional Mayor de San Marcos, Lima, Perú. An Fac Med. 2014;75(2):167-72.

4. Henriquez C, Guillen C, Bustamante B, Tello R. Micosis superficial en poblaciones selváticas: prevalencia en cuatro comunidades rurales: Santa Rosa de Tiocayu, Pamashto, Bello
Horizonte y Sachvacayoc. Folia Dermatol Peru. 2000;11(1):30-5.

5. Li JW-H, Vederas JC. Drug Discovery and Natural Products: End of an Era or an Endless Frontier? Science. 2009;325(5937):161-5.

6. Blanco EC, González JCM. El uso de la hoja de coca como manifestación cultural inmaterial. Criterio Juríd Garantista [Internet]. 2014 [citado el 3 de enero de 2016];6(11). Recuperado a partir de: http://revistas.fuac.edu.co/index. php/criteriojuridicogarantista/article/view/441

7. Chadwick DJ, Marsh J, others. Bioactive compounds from plants. [Internet]. John Wiley; 1990 [citado el 2 de enero de 2016]. Recuperado a partir de: http://www.cabdirect.org/ abstracts/19902302850.html

8. González-Guevara JL, González-Lavaut JA, Pino-Rodríguez S, García-Torres M, Carballo-González MT, Echemendia-Arana $\mathrm{OA}$, et al. Phytochemical screening and in vitro antiherpetic activity of four Erythroxylum species. Acta Farm Bonaer [Internet]. 2004 [citado el 2 de enero de 2016];23(4). Recuperado a partir de: http://www.latamjpharm.org/ trabajos/23/4/LAJOP_23_4_2_2_9M613X379D.pdf

9. González-García K, Gonzalez-Lavaut JA, González-Guevara J, Prieto-Gonzalez S. Género Erythroxylum: análisis de la información científica. Acta Farm Bonaer. 2005;24(2):284.

10. Lohezic F, Amoros M, Boustie J, Girre L. In-vitro Antiherpetic Activity of Erythroxylon laurifolium (Erythroxylaceae). Pharm Pharmacol Commun. 1999;5(3):249-53.

11. Marzouk B, Marzouk Z, Décor R, Mhadhebi L, Fenina N, Aouni M. Antibacterial and antifungal activities of several populations of Tunisian Citrullus colocynthis Schrad. immature fruits and seeds. J Med Mycol. 2010;20(3):179-84.

12. Cabieses Molina F. Mas sobre la coca. 2001 [citado el 2 de enero de 2016]; Recuperado a partir de: http://agris.fao.org/agrissearch/search.do?recordID=PE2001106311

13. Takarada K, Kimizuka R, Takahashi N, Honma K, Okuda K, Kato T. A comparison of the antibacterial efficacies of essential oils against oral pathogens. Oral Microbiol Immunol. 2004;19(1):614.

14. Cano C. Actividad antimicótica in vitro y elucidación estructural del aceite esencialde las hojas de Minthostachys mollis (muña). [Tesis para optar al Grado de Magister]. Facultad de Farmacia y Bioquímica UNMSM. Lima; 2007.

15. Cushnie TT, Lamb AJ. Antimicrobial activity of flavonoids. Int J Antimicrob Agents. 2005;26(5):343-56.

16. Jaitak V, Kaul VK, Kumar N, Singh B, Dhar J, Sharma OP, et al. New hopane triterpenes and antioxidant constituents from Potentilla fulgens. Nat Prod Commun. 2010;5(10):1561-6.

17. Ngane AN, Biyiti L, Zollo PA, Bouchet PH. Evaluation of antifungal activity of extracts of two Cameroonian Rutaceae: Zanthoxylum leprieurii Guill. et Perr. and Zanthoxylum xanthoxyloides Waterm. J Ethnopharmacol. 2000;70(3):335-42.

18. Galeotti F, Barile E, Curir P, Dolci M, Lanzotti V. Flavonoids from carnation (Dianthus caryophyllus) and their antifungal activity. Phytochem Lett. 2008;1(1):44-8.

19. Lock de Ugaz 0 . Investigación Fitoquímica-Métodos en el estudio de productos naturales. Lima-Perú Fondo Editor Pontif Univ Católica Perú [Internet]. 1988 [citado el 2 de enero de 2016]; Recuperado a partir de: http://www.sidalc.net/cgi-bin/ wxis.exe/?IsisScript=P-LIMxis\&method=post\&formato=2\&canti $\mathrm{dad}=1$ \&expresion=mfn=003681

20. Sathiamoorthy B, Gupta P, Kumar M, Chaturvedi AK, Shukla PK, Maurya R. New antifungal flavonoid glycoside from Vitex negundo. Bioorg Med Chem Lett. 2007;17(1):239-42.

21. Alarcón R, Flores RC, Ocampos S, Lucatti A, Galleguillo LF, Tonn C, et al. Flavonoids from Pterocaulon alopecuroides with antibacterial activity. Planta Med. 2008;74(12):1463. 
Efecto del extracto acuoso, ácido y alcohólico de las hojas secas de Erythroxylum coca var coca (coca) en Trichophyton rubrum, Trichophyton mentagrophytes, Microsporum canis y Candida albicans in vitro

22. Rojas R, Bustamante B, Bauer J, Fernández I, Albán J, Lock 0. Antimicrobial activity of selected Peruvian medicinal plants. $J$ Ethnopharmacol. 2003;88(2):199-204.

23. Giordani R, Trebaux J, Masi M, Regli P. Enhanced antifungal activity of ketoconazole by Euphorbia characias latex against Candida albicans. J Ethnopharmacol. 2001;78(1):1-5.

24. Eja ME, Arikpo GE, Enyi-Idoh KH, Etim SE, Etta HE. Efficacy of local herbal therapy in the management of dermatophytosis among primary school children in Cross River State, Southsouth Nigeria. Afr J Med Med Sci. 2009;38(2):135-41.

25. Sohn HY, Kun HS. Fungicidal effect of prenylated flavonol, papyriflavonol a, isolated from broussonetia papyrifera (L.) vent. against candida albicans. J Microbiol Biotechnol. 2010;20(10):1397-402.

26. Garcia VN, Gonzalez A, Fuentes M, Aviles M, Rios MY, Zepeda $\mathrm{G}$, et al. Antifungal activities of nine traditional Mexican medicinal plants. J Ethnopharmacol. 2003;87(1):85-8.

27. Mishra BB, Singh DD, Kishore N, Tiwari VK, Tripathi V. Antifungal constituents isolated from the seeds of Aegle marmelos. Phytochemistry. 2010;71(2):230-4.
28. Danelutte AP, Lago JHG, Young MCM, Kato MJ. Antifungal flavanones and prenylated hydroquinones from Piper crassinervium Kunth. Phytochemistry. 2003;64(2):555-9.

Fuentes de financiamiento:

Este artículo ha sido financiado por los autores.

Conflictos de interés:

Los autores declaran no tener ningún conflicto de interés.

\section{Correspondencia:}

Medalit Luna-Vílchez

Dirección: Jirón La Luna 275 Urb. San Roque, Santiago de Surco Teléfono: 953697536

Correo electrónico: medalv16@hotmail.com

Recibido: 31 de agosto de 2016

Aprobado: 22 de noviembre de 2016 